\title{
Enhanced Bubble Generation by an Air-Stimulated Co-Flow System
}

\author{
Javier Ruiz-Rus ${ }^{1}$, Rocío Bolaños-Jiménez ${ }^{1}$, Cándido Gutiérrez-Montes ${ }^{1}$, Alejandro Sevilla ${ }^{2}$, Carlos \\ Martínez-Bazán ${ }^{1}$ \\ ${ }^{1}$ Área de Mecánica de Fluidos, Departamento de Ingeniería Mecánica y Minera \\ Universidad de Jaén, 23071, Jaén, Spain \\ jrrus@ujaen.es; rbolanos@ujaen.es; cgmontes@ujaen.es; cmbazan@ujaen.es \\ ${ }^{2}$ Área de Mecánica de Fluidos, Departamento de Ingeniería Térmica y de Fluidos \\ Universidad Carlos III de Madrid, 28911, Leganés, Spain \\ asevilla@ing.uc3m.es
}

\section{Extended Abstract}

The cylindrical co-flow configuration, in which a gas stream discharges inside a laminar liquid jet, allows to generate monodisperse bubbles at frequencies much larger than those in the case of still liquid, at the same time that it avoids coalescence. Although the bubbling regime can be controlled by properly choosing both liquid and gas velocities, the size of the generated bubbles is strongly conditioned by the gas injector diameter [1]. Therefore, active methods to produce bubbles in a controlled way are still needed. Recently, the modulation of the liquid flow rate has been shown to be able to independently regulate both the generation frequency and bubble size in a planar co-flow configuration [2]. In contrast, here we present a method which acts over the gas phase in a cylindrical geometry, more extended in industrial applications.

Thus, the purpose of the present study is to experimentally analyse the mechanisms involved in the bubble formation dynamics in an axisymmetric co-flow configuration under harmonic pressure perturbations induced in the gas feeding line. To that aim, we report new bubbling experiments in a cylindrical gas-liquid co-flow with varying gas flow rate, forced by means of monochromatic pressure modulation of the gas phase. In order to establish the natural bubbling regimes, a short gas injection needle is used. Therefore, the pressure drop along the needle results comparable to the pressure fluctuations at the needle exit taking place during the bubbling process, causing the gas flow rate feeding the bubble to vary during the natural bubble formation cycle [3]. In addition, to generate the gas pressure perturbations, an acoustic forcing device, based on a loudspeaker placed in a specially designed gas feeding chamber where a constant gas flow rate enters upstream, has been incorporated into the gas line.

Thanks to this forcing process, the bubble generation frequency can be increased from the natural value, to a desired forcing frequency, provided that the amplitude of the pressure modulation is large enough. Moreover, given the periodicity of both the bubble production and the forcing signal, the volume of the generated bubbles almost coincides with that of the naturally grown bubbles at an instant equal to the forcing period. In order to describe the forcing process and its effects on the dynamics of the bubble generation, detailed observations of the time evolution of gas-liquid interface are performed by means of high-speed imaging, being the acquisitions synchronized with the pressure signal measured inside the chamber. Given the extremely fast nature of the bubble collapse and since the field of view has to be sufficient to capture the global bubbling process, the high-speed images are digitally analysed by means of a specially-developed sub-pixel detection algorithm, similar to that proposed in [4] to detect small oscillation amplitudes in perturbed liquid bridges.

\section{References}

[1] J. Rodríguez-Rodríguez, A. Sevilla, C. Martínez-Bazán, J. M. Gordillo, "Generation of microbubbles with applications to industry and medicine," Annu. Rev. Fluid Mech., vol. 47, pp. 405-429, 2015.

[2] J. Ruiz-Rus, R. Bolaños-Jiménez, C. Gutiérrez-Montes, A. Sevilla, C. Martínez-Bazán, "Controlled formation of bubbles in a planar co-flow configuration," Int. J. Multiph. Flow, vol. 89, pp. 69-80, 2017.

[3] J. M. Gordillo, A. Sevilla, C. Martínez-Bazán, "Bubbling in a co-flow at high Reynolds numbers," Phys. Fluids, vol. 19, no. 7, pp. 077102, 2007. 
[4] E. J. Vega, J. M. Montanero, J. Fernández, "On the precision of optical imaging to study free surface dynamics at high frame rates," Exp. Fluids, vol. 47, pp. 251-261, 2009. 